\title{
Truncated Weibull-G More Flexible and More Reliable than Beta-G Distribution
}

\author{
Hossein Najarzadegan ${ }^{1}$, Mohammad Hossein Alamatsaz ${ }^{1}$ \& Saied Hayati ${ }^{1}$ \\ ${ }^{1}$ Department of Statistics, University of Isfahan, Isfahan, Iran \\ Correspondence: M. H. Alamatsaz, Department of Statistics, University of Isfahan, Isfahan, Iran. \\ E-mail: alamatho@sci.ui.ac.ir
}

Received: May 28, 2017 Accepted: June 12, 2017 Online Published: July 21, 2017

doi:10.5539/ijsp.v6n5p1 URL: https://doi.org/10.5539/ijsp.v6n5p1

\begin{abstract}
Our purpose in this study includes introducing a new family of distributions as an alternative to beta-G (B-G) distribution with flexible hazard rate and greater reliability which we call Truncated Weibull-G (TW-G) distribution. We shall discuss several submodels of the family in detail. Then, its mathematical properties such as expansions, probability density function and cumulative distribution function, moments, moment generating function, order statistics, entropies, unimodality, stochastic comparison with the B-G distribution and stress-strength reliability function are studied. Moreover, we study shape of the density and hazard rate functions, and based on the maximum likelihood method, estimate parameters of the model. Finally, we apply the model to a real data set and compare B-G distribution with our proposed model.
\end{abstract}

Keywords: Entropy, Moment, Moment generating function, Stress-strength reliability function, Weibull distribution

\section{Introduction}

In the past decades, many authors have shown interest in obtaining new classes of probability distributions with higher flexibility in applications. Exponentiated exponential family by Gupta and Kundu (2001), exponentiated Weibull family by Nassar and Eissa (2003), exponentiated beta distribution by Nadarajah (2005a), exponentiated Pareto distribution by Nadarajah (2005b) and generalized Gumbel distribution by Cooray (2010) are examples of such interests.

Eugene, Lee and Famoye (2002) proposed a family of beta-G distributions by

$$
F(x)=\frac{1}{B(\beta, \alpha)} \int_{0}^{G(x)} t^{\beta-1}(1-t)^{\alpha-1} d t,
$$

where $\beta>0, \alpha>0, G(x)$ is a distribution function and $B(\beta, \alpha)=\int_{0}^{1} t^{\alpha-1}(1-t)^{\beta-1} d t$ is the beta function. Recently, several new distributions have been introduced following the proposed work by Eugene et al. (2002), including the betaGumble and beta-exponential distributions by Nadarajah and Kots $(2004,2006)$, respectively, beta-Weibull distribution by Lee, Famoye and Olumolade (2007), beta-generalized exponential distribution by Barreto-Souza, Santos and Cordeiro (2010), beta-modified Weibull distribution by Silva, Ortega and Cordeiro (2010), beta-Weibull-geometric distribution by Cordeiro, Silva and Ortega (2013), beta-generalized gamma distribution by Cordeiro et al. (2013), beta-lindley distribution by MirMostafaee, Mahdizadeh and Nadarajah (2015) and beta-generalized Marshall-Olkin-G family by Handique and Chakraborty (2016).

Weibull distribution plays an important role in Reliability Theory. In fact, Weibull distribution is the first candidate for modeling cases with monotone hazard rates but it is not a good candidate for modeling cases with non-monotone hazard rates. Thus, we can see several authors attempting to modify the Weibull distribution for applying in cases with nonmonotone hazard rates. The truncated Weibull distribution is one of such distributions with bathtub shaped hazard rate function. The truncated Weibull distribution has been applied in several engineering fields, partly because it has more flexible hazard rate than Weibull distribution.

As seen in Eq. (1), the baseline pdf is beta distribution defined on the interval $[0,1]$. This fact motivated us to introduce a new class of truncated Weibull-G distributions by inserting the truncated Weibull distribution with support $[0,1]$ into Eq. (1), instead of the beta distribution. Truncated Weibull-G distribution compared to Beta-G distribution is more reliable and has explicit forms for its cumulative distribution function (cdf) and hazard rate function (hrf).

Therefore, in Section 2, first propose the new truncated Weibull-G (TW-G) family of distributions. Density, hazard rate and quantile functions of this model are discussed in this section. In Section 3, its several submodels are studied. Several mathematical characteristics of the model such as cdf and pdf, moments and moment generating function, Renyi and Shannon entropies, order statistics, unimodal property and stress-stregnth reliability function for TW-G distributions are 
considered in Section 4. In this section, the new family are stochastically compared with the more classic B-G distinction and it is revealed that as a lifetime distribution it has larger reliability. We then describe shapes behavior of the pdf and hrf in Section 5. Maximum likelihood estimates and the observed information matrix are considered in Section 6. In last section, an application of several submodels to a real data are considered and they are compared to several corresponding submodels of beta-G distribution, where it is illustrated that our proposed distribution has better reliability than the beta-G distribution in (1).

\section{The New Family}

By replacing the truncated Weibull distribution with support $[0,1]$ with beta distribution in Eq. (1), we have

$$
F(x)=\int_{0}^{G(x)} \frac{\alpha \beta t^{\beta-1} \exp \left(-\alpha t^{\beta}\right)}{1-\exp (-\alpha)} d t=\frac{1-\exp \left(-\alpha G(x)^{\beta}\right)}{1-\exp (-\alpha)} . \quad \alpha>0, \beta>0
$$

Henceforth, we indicate a random variable $X$ with cdf (2) by $X \sim \mathrm{TW}-\mathrm{G}(\alpha, \beta)$, where $\alpha>0, \beta>0$ and it is called truncated Weibull-G distribution. Clearly, density function corresponding to (2) is denoted by

$$
f(x)=\frac{\alpha \beta G(x)^{\beta-1} \exp \left(-\alpha G(x)^{\beta}\right) g(x)}{1-\exp (-\alpha)},
$$

where $g$ is pdf corresponding to $G$. Thus, the hrf of $X$ becomes

$$
h(x)=\frac{\alpha \beta G(x)^{\beta-1} \exp \left(-\alpha G(x)^{\beta}\right) g(x)}{\exp \left(-\alpha G(x)^{\beta}\right)-\exp (-\alpha)}
$$

The quantile function (qf) of the TW-G distribution can be generated by inverting cdf (2), as

$$
Q(u)=G^{-1}\left(\left(\log \left(\frac{1}{1-u\left(1-e^{-\alpha}\right)}\right)^{\frac{1}{\alpha}}\right)^{\frac{1}{\beta}}\right)
$$

where $G^{-1}$ is the quantile function of the parent distribution $G$, and $u \in(0,1)$. Recall that

$$
\begin{array}{ll}
\log (1-x)=-\sum_{j=0}^{\infty} \frac{x^{j+1}}{j+1}, & |x|<1, \\
(1-x)^{r}=\sum_{j=0}^{\infty}\left(\begin{array}{l}
r \\
j
\end{array}\right)(-1)^{j} x^{j}, & |x|<1,
\end{array}
$$

and an equation which is defined by Gradshteyn and Ryzhik (2007), we have

$$
\left(\sum_{j=0}^{\infty} a_{j} x^{j}\right)^{n}=\sum_{j=0}^{\infty} d_{n, j} x^{j}
$$

where $n \in N=1,2, \ldots, d_{n, j}=\left(j a_{0}\right)^{-1} \sum_{m=1}^{j}[m(n+1)-j] a_{m} d_{n, j-m}$ and $d_{n, 0}=a_{0}^{n}$. Thus, qf Q in (5) can be expanded as 
follows:

$$
\begin{aligned}
Q(u) & =G^{-1}\left(\left(\log \left(1-u\left(1-e^{-\alpha}\right)\right)^{\frac{-1}{\alpha}}\right)^{\frac{1}{\beta}}\right), \\
& =G^{-1}\left(\left(\frac{1}{\alpha}\right)^{\frac{1}{\beta}}\left(-\log \left(1-u\left(1-e^{-\alpha}\right)\right)\right)^{\frac{1}{\beta}}\right), \\
& =G^{-1}\left[\left(\frac{1}{\alpha}\right)^{\frac{1}{\beta}}\left(1-\left[1+\log \left[1-u\left(1-e^{-\alpha}\right)\right]\right]\right)^{\frac{1}{\beta}}\right], \\
& =G^{-1}\left[\left(\frac{1}{\alpha}\right)^{\frac{1}{\beta}} \sum_{j=0}^{\infty}\left(\begin{array}{c}
\frac{1}{\beta} \\
j
\end{array}\right)(-1)^{j}\left(1+\log \left[1-u\left(1-e^{-\alpha}\right)\right]\right)^{j}\right], \\
& =G^{-1}\left[\left(\frac{1}{\alpha}\right)^{\frac{1}{\beta}} \sum_{j=0}^{\infty}\left(\begin{array}{c}
\frac{1}{\beta} \\
j
\end{array}\right)(-1)^{j} \sum_{k=0}^{j}\left(\begin{array}{l}
j \\
k
\end{array}\right)\left(\log \left[1-u\left(1-e^{-\alpha}\right)\right]\right)^{j}\right], \\
& =G^{-1}\left[\left(\frac{1}{\alpha}\right)^{\frac{1}{\beta}} \sum_{j=0}^{\infty} \sum_{k=0}^{j}\left(\begin{array}{c}
\frac{1}{\beta} \\
j
\end{array}\right)\left(\begin{array}{l}
j \\
k
\end{array}\right)(-1)^{j}\left(\log \left[1-u\left(1-e^{-\alpha}\right)\right]\right)^{j}\right], \\
& =G^{-1}\left[\left(\frac{1}{\alpha}\right)^{\frac{1}{\beta}} \sum_{j=0}^{\infty} \sum_{k=0}^{j}\left(\begin{array}{c}
\frac{1}{\beta} \\
j
\end{array}\right)\left(\begin{array}{l}
j \\
k
\end{array}\right)(-1)^{j}\left(-u \sum_{l=0}^{\infty} \frac{u^{l}\left(1-e^{-\alpha}\right)^{l+1}}{l+1}\right)^{j}\right], \\
& =G^{-1}\left[\left(\frac{1}{\alpha}\right)^{\frac{1}{\beta}} \sum_{j, l=0}^{\infty} \sum_{k=0}^{j}\left(\begin{array}{l}
\frac{1}{\beta} \\
j
\end{array}\right)\left(\begin{array}{l}
j \\
k
\end{array}\right) u^{l+j} d_{j, l}\right],
\end{aligned}
$$

where $d_{j, l}=\left(l\left(1-e^{-\alpha}\right)\right)^{-1} \sum_{m=1}^{l}[m(j+1)-l] \frac{\left(1-e^{-\alpha}\right)^{m+1}}{m+1} d_{j, l-m}$ for $l>0$ and $d_{j, 0}=1$. By substituting proper values for $u$ in (9), we can obtain quantiles of interest. As a special case, the median of $X$ is given by

$$
\operatorname{Median}(X)=Q_{G}\left(\left(\log \left(\frac{1}{1-\frac{1}{2}\left(1-e^{-\alpha}\right)}\right)^{\frac{1}{\alpha}}\right)^{\frac{1}{\beta}}\right) .
$$

Hence, for simulating random variable $X$ from uniform random variable $U$ on the unit interval $(0,1)$, we have

$$
X=Q_{G}\left(\left(\log \left(\frac{1}{1-U\left(1-e^{-\alpha}\right)}\right)^{\frac{1}{\alpha}}\right)^{\frac{1}{\beta}}\right)
$$

\section{Special Submodels}

In the present section, we introduce six interesting submodels of the TW-G distribution.

\section{i) TW-Uniform distribution(TW-U):}

Let the parent distribution $G$ be a uniform distribution on the interval $(0, \delta), \delta>0$, i.e., $G(x, \delta)=\frac{x}{\delta}, x \in(0, \delta)$. Then, We have $g(x, \delta)=\frac{1}{\delta}$ and, consequently, the cdf and pdf of a TW-U are given by

$$
\begin{array}{ll}
F_{T W-U}(x, \alpha, \beta, \delta)=\frac{1-\exp \left(-\alpha\left(\frac{x}{\delta}\right)^{\beta}\right)}{1-\exp (-\alpha)} & 0<x<\delta . \\
f_{T W-U}(x, \alpha, \beta, \delta)=\frac{\alpha \beta\left(\frac{x}{\delta}\right)^{\beta-1} \exp \left(-\alpha\left(\frac{x}{\delta}\right)^{\beta}\right)\left(\frac{1}{\delta}\right)}{1-\exp (-\alpha)} & 0<x<\delta,
\end{array}
$$

\section{ii) TW-Weibull distribution(TW-W):}

Let the parent distribution $G$ be a Weibull distribution, i.e., $G(x, \lambda, \gamma)=1-\exp \left(-\lambda x^{\gamma}\right)$ for $\lambda, \gamma>0$. Then, we have $g(x, \lambda, \gamma)=\lambda \gamma x^{\gamma-1} \exp \left(-\lambda x^{\gamma}\right)$, and, consequently, the cdf and pdf of a TW-W are given by

$$
\begin{array}{ll}
F_{T W W}(x, \alpha, \beta, \lambda, \gamma)=\frac{1-\exp \left(-\alpha\left(1-\exp \left(-\lambda x^{\gamma}\right)\right)^{\beta}\right)}{1-\exp (-\alpha)} & x>0, \\
f_{T W W}(x)=\frac{\alpha \beta\left(1-\exp \left(-\lambda x^{\gamma}\right)\right)^{\beta-1} \exp \left(-\alpha\left(1-\exp \left(-\lambda x^{\gamma}\right)\right)^{\beta}\right) \lambda \gamma x^{\gamma-1} \exp \left(-\lambda x^{\gamma}\right)}{1-\exp (-\alpha)} & x>0 .
\end{array}
$$


iii) TW-Logisticl distribution(TW-L):

Let the parent distribution $G$ be a logistic distribution, i.e., $G(x, \eta)=(1+\exp (-\eta x))^{-1}$ for $\eta>0$. Then, we have $g(x, \eta)=\eta \exp (-\eta x)(1+\exp (-\eta x))^{-2}$, and, consequently, the cdf and pdf of a TW-L are given by

$$
\begin{gathered}
F_{T W L}(x, \alpha, \beta, \eta)=\frac{1-\exp \left(-\alpha(1+\exp -\eta x)^{-\beta}\right)}{1-\exp (-\alpha)} \quad x>0, \\
f_{T W L}(x)=\frac{\alpha \beta(1+\exp (-\eta x))^{1-\beta} \exp \left(-\alpha(1+\exp (-\eta x))^{-\beta}\right) \eta \exp (-\xi x)(1+\exp (-\eta x))^{-2}}{1-\exp (-\alpha)} \quad x>0 .
\end{gathered}
$$

\section{iv) TW-Log Logisticl distribution(TW-LL):}

Let the parent distribution $G$ be a $\log$ logistic distribution, i.e., $G(x, s, c)=1-\left(1+\left(\frac{x}{s}\right)^{c}\right)^{-1}$ with parameters $s>0$ and $c>0$. Then, we have $g(x, s, c)=c s^{-c} x^{c-1}\left(1+\left(\frac{x}{s}\right)^{c}\right)^{-2}$, and, consequently, the cdf and pdf of a TW-LL are given by

$$
\begin{aligned}
& F_{T W L L}(x, \alpha, \beta, s, c)=\frac{1-\exp \left(-\alpha\left(1-\left(1+\left(\frac{x}{s}\right)^{c}\right)^{-1}\right)^{\beta}\right)}{1-\exp (-\alpha)} \quad x>0, \\
& f_{T W L L}(x)=\frac{\alpha \beta\left(1-\left(1+\left(\frac{x}{s}\right)^{c}\right)^{-1}\right)^{\beta-1} \exp \left(-\alpha\left(1-\left(1+\left(\frac{x}{s}\right)^{c}\right)^{-1}\right)^{\beta}\right) c s^{-c} x^{c-1}\left(1+\left(\frac{x}{s}\right)^{c}\right)^{-2}}{1-\exp (-\alpha)} \quad x>0 .
\end{aligned}
$$

\section{v) TW-Burr XII distribution(TW-BXII):}

Let the parent distribution $G$ be a Burr XII distribution, i.e., $G(x, s, c, k)=1-\left(1+\left(\frac{x}{s}\right)^{c}\right)^{-k} ; s, c, k>0$. Then, we have $g(x, s, c, k)=c k s^{-c} x^{c-1}\left(1+\left(\frac{x}{s}\right)^{c}\right)^{-k-1}$, and, consequently, the cdf and pdf of a TW-BXII are given by

$$
\begin{array}{ll}
F_{T W B X I I}(x, \alpha, \beta, s, c, k)=\frac{1-\exp \left(-\alpha\left(1-\left(1+\left(\frac{x}{s}\right)^{c}\right)^{-k}\right)^{\beta}\right)}{1-\exp (-\alpha)} & x>0, \\
f_{T W B X I I}(x)=\frac{\alpha \beta\left(1-\left(1+\left(\frac{x}{s}\right)^{c}\right)^{-k}\right)^{\beta-1} \exp \left(-\alpha\left(1-\left(1+\left(\frac{x}{s}\right)^{c}\right)^{-k}\right)^{\beta}\right) c k s^{-c} x^{c-1}\left(1+\left(\frac{x}{s}\right)^{c}\right)^{-k-1}}{1-\exp (-\alpha)} & x>0 .
\end{array}
$$

\section{vi) TW-Normall distribution(TW-N):}

Let the parent distribution $G$ be a Normal distribution, i.e., $G(x, \mu, \sigma)=\Phi\left[\frac{(x-\mu)}{\sigma}\right] ; \mu \in \mathbf{R}, \sigma^{2}>0$. Then, we have $g(x, \mu, \sigma)=\frac{\phi\left[\frac{(x-\mu)}{\sigma}\right]}{\sigma}$ and, consequently, the cdf and pdf of a TW-N are given by

$$
\begin{array}{ll}
F_{T W N}(x, \alpha, \beta, \mu, \sigma)=\frac{1-\exp \left(-\alpha\left(\Phi\left[\frac{(x-\mu)}{\sigma}\right]\right)^{\beta}\right)}{1-\exp (-\alpha)} & x>0, \\
f_{T W N}(x)=\frac{\alpha \beta\left(\Phi\left[\frac{(x-\mu)}{\sigma}\right]\right)^{\beta-1} \exp \left(-\alpha\left(\Phi\left[\frac{(x-\mu)}{\sigma}\right]\right)^{\beta}\right) \frac{\phi\left[\frac{(x-\mu)}{\sigma}\right]}{\sigma}}{1-\exp (-\alpha)} & x>0 .
\end{array}
$$

\section{Mathematical Properties}

Here, we present several mathematical properties of the our new proposed distribution.

\subsection{Expansion of the $c d f$ and $p d f$ of $T W-G$}

Here, we express expansion of cdf (2) by applying the power series expansion for the exponential function as follows,

$$
F(x)=\frac{1}{1-\exp (-\alpha)}\left[\sum_{i=1}^{\infty} \frac{(-1)^{i} \alpha^{i}}{i !} G(x)^{i \beta}\right] .
$$

Since,

$$
G(x)^{i \beta}=\sum_{j=0}^{\infty}\left(\begin{array}{c}
i \beta \\
j
\end{array}\right)(-1)^{j}(1-G(x))^{j},
$$


and

$$
[1-G(x)]^{j}=\sum_{k=0}^{j}\left(\begin{array}{l}
j \\
k
\end{array}\right)(-1)^{k} G(x)^{k},
$$

where,

$$
\left(\begin{array}{c}
i \beta \\
j
\end{array}\right)=\frac{(i \beta)(i \beta-1) \ldots(i \beta-j+1)}{j !},
$$

we obtain

$$
\begin{aligned}
F(x)=\frac{1}{1-\exp (-\alpha)} & {\left[\sum_{i=1}^{\infty} \frac{(-1)^{i} \alpha^{i}}{i !} \sum_{j=0}^{\infty}\left(\begin{array}{c}
i \beta \\
j
\end{array}\right) \sum_{k=0}^{j}\left(\begin{array}{l}
j \\
k
\end{array}\right)(-1)^{j+k} G(x)^{k}\right], } \\
& =\sum_{k=0}^{\infty}\left[\sum_{i=1}^{\infty} \sum_{j=k}^{\infty} \frac{(-1)^{i} \alpha^{i}}{i !(1-\exp (-\alpha))}\left(\begin{array}{c}
i \beta \\
j
\end{array}\right)\left(\begin{array}{l}
j \\
k
\end{array}\right)(-1)^{j+k}\right] G(x)^{k} .
\end{aligned}
$$

Therefore, we can write $F(x)$ as

$$
F(x)=\sum_{k=0}^{\infty} b_{k} F_{k}^{*}(x),
$$

where

$$
b_{k}=\sum_{i=1}^{\infty} \sum_{j=k}^{\infty} \frac{(-1)^{i+j+k} \alpha^{i}}{i !(1-\exp (-\alpha))}\left(\begin{array}{c}
i \beta \\
j
\end{array}\right)\left(\begin{array}{l}
j \\
k
\end{array}\right),
$$

and $F_{k}^{*}(x)=G(x)^{k}$ (for $k>0$ ) is the exponentiated- $G$ (exp-G) cdf with power parameter $k$ and $F_{0}^{*}(x)=1$. By simple differentiation of Eq. (11), we can write

$$
f(x)=\sum_{k=0}^{\infty} b_{k+1} f_{k+1}^{*}(x),
$$

where $f_{k+1}^{*}(x)=(k+1) g(x) G(x)^{k}$ is the exp-G density function with parameter(k+1). Now, since (12) is a mixture of exp$\mathrm{G}$ densities, thus, we can derive certain mathematical attributes of the TW-G model from the exp-G distribution existing in the literature for exp-G distributions such as ordinary and incomplete moments and moment generating function (mgf) which have been considered by Mudholkar, Srivastava and Freimer (1995), Gupta and Kundu (1999), Nadarajah and Kots (2006) and others.

\subsection{Moments}

The $n$th moment of $X$ can be obtioned from (12) as

$$
E\left(X^{n}\right)=\sum_{k=0}^{\infty} b_{k+1} E\left(Y_{k}^{n}\right),
$$

where $Y_{k}$ is a random variable with exp-G density function $f_{k+1}^{*}(x)$. Nadarajah and Kots (2006) obtained ordinary moments of some well-known exp-G models. such as exp-Gamma, exp-Weibull, exp-Gumbel and exp-Frechet distributions. These can be used to produce $E\left(X^{n}\right)$ in (13). We can also obtain the ordinary moment $E\left(X^{n}\right)$ using qf $G^{-1}(u)$ as

$$
E\left(X^{n}\right)=\sum_{k=0}^{\infty}(k+1) b_{k+1} \xi_{n}(k),
$$

where

$$
\xi_{n}(k)=\int_{-\infty}^{\infty} x^{n} G(x)^{k} g(x) d x=\int_{0}^{1}\left[G^{-1}(u)\right]^{n} u^{k} d u
$$

Hence, the ordinary moments of TW-G distributions can be obtained from Eqs. (14) and (15). Cordeiro and Nadarajah (2011) determined $\xi_{n}(k)$ for some well-known distributions. We also, compute, here, $\xi_{n}(k)$ for three cases as examples. 
Example. 1. For the TW-W distribution by integral equation

$$
\int_{0}^{1} x^{\alpha-1} \log ^{n}(x) d x=(-1)^{n} \alpha^{-n-1} n ! \quad \alpha>0,
$$

we have

$$
\xi_{n}(k)=\frac{1}{\lambda^{\frac{n}{\gamma}}} \sum_{j=0}^{\infty} \sum_{q=0}^{j} \sum_{l=0}^{k}\left(\begin{array}{l}
k \\
l
\end{array}\right) \frac{\left(\frac{n}{\gamma}\right)^{(j)}(-1)^{j+l+q}}{(j-q) !(l+1)^{q+1}},
$$

where $x^{(k)}=x(x+1) \ldots(x+k-1)$.

Example. 2. For the TW-L distribution by

$$
\frac{\partial^{n}}{\partial t^{n}}\left[\int_{0}^{1} e^{(t+k) \log x-t \log (1-x)} d x\right]_{t=0}=\int_{0}^{1} x^{k}\left(\log \left(\frac{x}{1-x}\right)\right)^{n},
$$

we have

$$
\xi_{n}(k)=\frac{1}{\eta^{n}} \frac{\partial^{n}}{\partial t^{n}}(B(t+k+1,1-t))_{t=0}
$$

where $B(\alpha, \beta)=\int_{0}^{1} x^{\alpha-1}(1-x)^{\beta-1} d x$.

Example. 3. For the TW-LL distribution we have

$$
\xi_{n}(k)=s^{n} \sum_{j=0}^{\infty} \frac{\left(\frac{n}{c}\right)^{(j)}}{j !\left(\frac{n}{c}+j+k+1\right)} .
$$

Due to the important role of incomplete moments in measuring indices of income inequality such as Lorenz and Bonferroni curves and the associated Gini index, we need to determine the $n$th incomplete moments as follows.

$$
m_{n}(y)=\sum_{k \geq 0}(k+1) b_{k+1} H_{n, k}(G(y))
$$

where

$$
H_{n, k}(x)=\int_{0}^{x}\left[G^{-1}(u)\right]^{n} u^{k} d u .
$$

We can compute (21) for most baselina $G$ distributions as shown in the following examples.

Example. 4. For the TW-W distribution by (8) we have

$$
H_{n, k}(G(y))=\frac{1}{\lambda^{\frac{n}{\gamma}}} \sum_{j, l=0}^{\infty} \sum_{q=0}^{j}\left(\begin{array}{l}
\frac{n}{\gamma} \\
j
\end{array}\right)\left(\begin{array}{l}
j \\
q
\end{array}\right)(-1)^{j+q} \frac{[G(y)]^{k+q+l+1}}{k+q+l+1} d_{q, l},
$$

where $d_{q, l}=l^{-1} \sum_{m=0}^{l}[m(q+1)-l] \frac{d_{q, l-m}}{m+1}$ and $G$ is Weibull distribution.

Example. 5. For the TW-L distribution we have

$$
H_{n, k}(G(y))=\frac{1}{\eta^{n}} \frac{\partial^{n}}{\partial t^{n}}\left(B_{G(y)}(t+k+1,1-t)\right)_{t=0},
$$

where $B_{x}(\alpha, \beta)=\int_{0}^{x} t^{\alpha-1}(1-t)^{\beta-1} d t$ and $G$ is logistic distribution.

Example. 6. For the TW-LL distribution, where $G$ is $\log \operatorname{logistic}$ distribution by (16) we have

$$
H_{n, k}(G(y))=\sum_{j=0}^{\infty} \frac{s^{n}\left(\frac{n}{c}\right)^{(j)}[G(y)]^{k+j+\frac{n}{c}+1}}{j !\left(k+j+\frac{n}{c}+1\right)} .
$$

In what follows, the probability weighted moments (PWMs) of a TW-G $(\alpha, \beta)$ random variable $X$ is obtained. In particular, when the inverse of a distribution does not have a closed form or maximum likelihood estimates are unavailable or difficult to compute, PWMs moments can be used to estimate the parameters. They may also be used as starting values 
for maximum likelihood estimates. The PWMs are expectations of multiplication of two certain functions of a random variable $X$ defined as

$$
\tau_{n, r}=E\left[X^{n} F(X)^{r}\right]=\int_{-\infty}^{\infty} x^{n} F(x)^{r} f(x) d x .
$$

Thus, by using (8),(11) and (12), we have

$$
\begin{aligned}
\tau_{n, r} & =\int_{-\infty}^{\infty} x^{n} F(x)^{r} f(x) d x=\int_{-\infty}^{\infty} x^{n}\left(\sum_{t=0}^{\infty} b_{t} G(x)^{t}\right)^{r} \sum_{k=0}^{\infty} b_{k+1}(k+1) g(x) G(x)^{k} d x, \\
& =\sum_{t, k=0}^{\infty} b_{k+1}(k+1) d_{r, t} \xi_{n}(k+t),
\end{aligned}
$$

where $d_{r, t}=\left(t b_{0}\right)^{-1} \sum_{m=1}^{t}[m(t+1)-t] b_{m} d_{r, t-m}$ and $d_{r, 0}=b_{0}^{r}$. Hence, we can derive closed-form expressions for (22) by determing $\xi_{n}(k+t)$ for different parent distributions. Indeed, we have calculated $\xi_{n}(k)$ for TW-W, TW-L and TW-LL in Examples 1, 2 and 3.

\subsection{Moment Generating Function}

Let $M_{X}(t)=E\left(e^{t X}\right)$ be the moment generating function (mgf) of $X$. Then, by (12), we have

$$
M_{X}(t)=\sum_{k=0}^{\infty} b_{k+1} E\left(e^{t Y_{k}}\right) .
$$

Hence, we can obtain $M_{X}(t)$ from $M_{Y_{k}}(t)$. The mgf of $X$ can also be determined by qf $G^{-1}(u)$ as

$$
M_{X}(t)=\sum_{k=0}^{\infty}(k+1) b_{k+1} \rho(t, k),
$$

where

$$
\rho(t, k)=\int_{-\infty}^{\infty} e^{t x} G(x)^{k} g(x) d x=\int_{0}^{1} e^{t G^{-1}(u)} u^{k} d u .
$$

We can obtain the mgfs of the special cases (i) to (vi) from Eqs. (24) and (25). By using integral equation

$$
\int_{0}^{1} x^{\alpha-1} \log ^{\sigma}\left(\frac{1}{x}\right) d x=\alpha^{-\sigma-1} \Gamma(\sigma+1) \quad \alpha>0 ; \sigma>-1,
$$

and (16), the integral (25) for the TW-W, TW-L and TW-LL distributions are given by

$$
\begin{gathered}
\rho(t, k)=\sum_{j=0}^{\infty} \sum_{l=0}^{k}\left(\begin{array}{l}
k \\
l
\end{array}\right) \frac{t^{j}(-1)^{l}}{j ! \lambda^{\frac{j}{\gamma}}(l+1)^{\frac{j}{\gamma}+1}} \Gamma\left(\frac{j}{\gamma}+1\right), \\
\rho(t, k)=\sum_{j=0}^{\infty} \frac{\left(\frac{t}{\eta}\right)^{(j)}}{j !\left(j+k+\frac{t}{\eta}+1\right)}
\end{gathered}
$$

and

$$
\rho(t, k)=\sum_{j=0}^{\infty} \sum_{l=0}^{\infty} \frac{(t s)^{j}\left(\frac{j}{c}\right)^{(l)}}{j ! l !\left(l+k+\frac{j}{c}+1\right)},
$$

respectively.

\subsection{Renyi and Shannon Entropies}

An entropy is a measure of uncertainty of a random variable X. It is an important tool in Science and Engineering. There are several different entropy and information indices from which Renyi and Shannon entropies are most popular. The Renyi entropy for a random variable $X$ with pdf $f($.) is defined as

$$
I_{F}(\delta)=\frac{1}{1-\delta} \log \int_{-\infty}^{\infty} f(x)^{\delta} d x,
$$


for $\delta>0$ and $\delta \neq 1$. Shannon entropy is the expectation of $\{-\log [f(X)]\}$ where $f$ is a density function of a random variable $X$, which is a special case of Renyi entropy when $\delta \uparrow 1$. Now we shall compute the Renyi and Shannon entropies for a TW-G random variable $X$. Since

$$
\begin{aligned}
& f(x)^{\delta}=\left(\frac{\alpha \beta}{1-e^{-\alpha}}\right)^{\delta} e^{-\delta \alpha G(x)^{\beta}} G(x)^{\delta(\beta-1)} g(x)^{\delta}, \\
& =\left(\frac{\alpha \beta}{1-e^{-\alpha}}\right)^{\delta} g(x)^{\delta} \sum_{i=0}^{\infty} \frac{(-1)^{i}(\delta \alpha)^{i}}{i !}(G(x))^{i \beta}(G(x))^{\delta(\beta-1)}, \\
& =\left(\frac{\alpha \beta}{1-e^{-\alpha}}\right)^{\delta} g(x)^{\delta} \sum_{i=0}^{\infty} \frac{(-1)^{i}(\delta \alpha)^{i}}{i !}(G(x))^{i \beta+\delta(\beta-1)} \text {, } \\
& =\left(\frac{\alpha \beta}{1-e^{-\alpha}}\right)^{\delta} g(x)^{\delta} \sum_{i=0}^{\infty} \frac{(-1)^{i}(\delta \alpha)^{i}}{i !} \sum_{j=0}^{\infty}(-1)^{j}\left(\begin{array}{c}
i \beta+\delta(\beta-1) \\
j
\end{array}\right)(1-G(x))^{j}, \\
& =\left(\frac{\alpha \beta}{1-e^{-\alpha}}\right)^{\delta} g(x)^{\delta} \sum_{i=0}^{\infty} \frac{(-1)^{i}(\delta \alpha)^{i}}{i !} \sum_{j=0}^{\infty}(-1)^{j}\left(\begin{array}{c}
i \beta+\delta(\beta-1) \\
j
\end{array}\right) \sum_{k=0}^{j}\left(\begin{array}{l}
j \\
k
\end{array}\right)(-1)^{k} G(x)^{k} \text {, } \\
& =\left(\frac{\alpha \beta}{1-e^{-\alpha}}\right)^{\delta} g(x)^{\delta} \sum_{i=0}^{\infty} \frac{(-1)^{i}(\delta \alpha)^{i}}{i !} \sum_{k=0}^{\infty} \sum_{j=k}^{\infty}(-1)^{j+k}\left(\begin{array}{c}
i \beta+\delta(\beta-1) \\
j
\end{array}\right)\left(\begin{array}{l}
j \\
k
\end{array}\right) G(x)^{k}, \\
& =\sum_{k=0}^{\infty}\left[\sum_{i=0}^{\infty} \sum_{j=k}^{\infty}\left(\frac{\alpha \beta}{1-e^{-\alpha}}\right)^{\delta} \frac{(-1)^{j+k+i}(\delta \alpha)^{i}}{i !}\left(\begin{array}{c}
i \beta+\delta(\beta-1) \\
j
\end{array}\right)\left(\begin{array}{l}
j \\
k
\end{array}\right)\right] G(x)^{k} g(x)^{\delta} \text {, }
\end{aligned}
$$

according to Eq. (28), we have

$$
I_{F}(\delta)=\frac{1}{1-\delta} \log \left[\sum_{k=0}^{\infty} S_{k} D_{\delta}(k)\right]
$$

where

$$
D_{\delta}(k)=\int_{-\infty}^{\infty} g(x)^{\delta} G(x)^{k} d x=\int_{0}^{1}\left[g\left(G^{-1}(u)\right)\right]^{\delta-1} u^{k} d u
$$

and

$$
S_{k}=\sum_{i=0}^{\infty} \sum_{j=k}^{\infty}\left(\frac{\alpha \beta}{1-e^{-\alpha}}\right)^{\delta} \frac{(-1)^{j+k+i}(\delta \alpha)^{i}}{i !}\left(\begin{array}{c}
i \beta+\delta(\beta-1) \\
j
\end{array}\right)\left(\begin{array}{l}
j \\
k
\end{array}\right) .
$$

Practically, it is sufficient to obtain the integral equation (30). Now, for the TW-W, TW-L and TW-LL distributions, the integral equation (30) turns out to be

$$
D_{\delta}(k)=\lambda^{\frac{\delta-1}{\gamma}} \gamma^{\delta-1} \Gamma\left(\frac{(\gamma-1)(\delta-1)}{\gamma}+1\right) \sum_{p=0}^{k}(-1)^{p}(\delta+p)^{-\frac{(\gamma-1)(\delta-1)}{\gamma}-1}\left(\begin{array}{l}
k \\
p
\end{array}\right),
$$

where $\gamma \geq \frac{\delta-1}{\delta}$,

$$
D_{\delta}(k)=\eta^{\delta-1} B(k+\delta, \delta)
$$

and

$$
D_{\delta}(k)=\left(\frac{c}{s}\right)^{\delta-1} B\left(\frac{(c-1)(\delta-1)}{c}+k+1,(\delta-1)\left(1+\frac{1}{c}\right)+1\right),
$$

respectively.

Thus, for the Shannon entropy we have

$$
E(-\log f(X))=-\log (\alpha \beta)+\log \left(1-e^{-\alpha}\right)+\alpha E\left(G^{\beta}(X)\right)-(\beta-1) E(\log G(X))-E(\log g(X)) .
$$

Based on Eq. (6), we can write

$$
E\left(G^{\beta}(X)\right)=\sum_{k=0}^{\infty} b_{k+1}(k+1) \int_{-\infty}^{\infty} g(x) G^{\beta+k}(x) d x=\sum_{k=0}^{\infty} \frac{b_{k+1}(k+1)}{\beta+k+1},
$$




$$
E(\log G(X))=\sum_{k=0}^{\infty} b_{k+1}(k+1) \int_{-\infty}^{\infty}(\log G(x)) G^{k}(x) g(x) d x=-\sum_{k=0}^{\infty} \frac{b_{k+1}}{(k+1)}
$$

and

$$
E(\log g(X))=\sum_{k=0}^{\infty} b_{k+1}(k+1) \int_{-\infty}^{\infty}(\log g(x)) G^{k}(x) g(x) d x=\sum_{k=0}^{\infty} b_{k+1}(k+1) \mathcal{W}_{k}
$$

where

$$
\mathcal{W}_{k}=\int_{0}^{1} \log \left(g\left(G^{-1}(u)\right)\right) u^{k} d u .
$$

The closed-form expressions for (32) can be obtained for deriving the Shannon entropy as shown in the following examples. For the TW-W distribution, using (6) and integral equation

$$
\int_{0}^{1} \log \log \frac{1}{x} x^{\mu-1} d x=-\frac{1}{\mu}(C+\log \mu), \quad[\mu>0, C=0.577215 \ldots]
$$

we obtain

$$
\mathcal{W}_{k}=\frac{\log \left(\lambda^{\frac{1}{\gamma}} \gamma\right)}{k+1}+\frac{\gamma-1}{\gamma} \sum_{l=0}^{k} \frac{(-1)^{l+1}}{l+1}[C+\log (l+1)]\left(\begin{array}{l}
k \\
l
\end{array}\right)+\sum_{p=0}^{k} \frac{(-1)^{p+1}}{(p+1)^{2}}\left(\begin{array}{l}
k \\
p
\end{array}\right) .
$$

For the TW-L distribution we have

$$
\mathcal{W}_{k}=\frac{\log \eta}{k+1}+\sum_{l=0}^{k} \frac{(-1)^{l+1}}{(l+1)^{2}}\left(\begin{array}{l}
k \\
l
\end{array}\right)-\frac{1}{(k+1)^{2}} .
$$

Finally, for the TW-LL distribution we have

$$
\mathcal{W}_{k}=\frac{\log \left(\frac{c}{s}\right)}{(k+1)}-\frac{c-1}{c(k+1)^{2}}+\left(\frac{c+1}{c}\right) \sum_{l=0}^{k} \frac{(-1)^{l+1}}{(l+1)^{2}}\left(\begin{array}{l}
k \\
l
\end{array}\right) \text {. }
$$

\subsection{Order Statistics}

Order statistics are important tools especially in non-parametric statistics and inference. Here, we shall consider order statistics properties of the class of TW-G distributions. Suppose that $X_{1}, X_{2}, \ldots, X_{n}$ is a random sample from the TW-G distribution and $X_{i: n}$ is the corresponding $i$ th order statistic. Then, we can express the pdf of $X_{i: n}$ as:

$$
\begin{aligned}
f_{i: n}(x) & =\frac{n !}{(i-1) !(n-i) !} f(x) F(x)^{i-1}(1-F(x))^{(n-i)}, \\
& =\frac{n !}{(i-1) !(n-i) !} \sum_{j=0}^{n-i}(-1)^{j}\left(\begin{array}{c}
n-i \\
j
\end{array}\right) f(x) F(x)^{j+i-1}, \\
& =\frac{n !}{(i-1) !(n-i) !} \sum_{j=0}^{n-i}(-1)^{j}\left(\begin{array}{c}
n-i \\
j
\end{array}\right)\left[\sum_{r=0}^{\infty} b_{r+1}(r+1) g(x) G(x)^{r}\right]\left[\sum_{k=0}^{\infty} b_{k} G(x)^{k}\right]^{i+j-1},
\end{aligned}
$$

and since by (8)

$$
\left[\sum_{k=0}^{\infty} b_{k} G(x)^{k}\right]^{i+j-1}=\sum_{k=0}^{\infty} d_{i+j-1, k} G(x)^{k}
$$

where $d_{i+j-1, k}=\left(k b_{0}\right)^{-1} \sum_{m=1}^{k}[m(i+j-1)-k] b_{m} d_{i+j-1, k-m}$ and $d_{i+j-1,0}=b_{0}^{i+j-1}$, we obtain

$$
\begin{aligned}
f_{i: n}(x) & =\frac{n !}{(i-1) !(n-i) !} \sum_{j=0}^{n-i}(-1)^{j}\left(\begin{array}{c}
n-i \\
j
\end{array}\right)\left[\sum_{r=0}^{\infty} b_{r+1}(r+1) g(x) G(x)^{r}\right]\left[\sum_{k=0}^{\infty} d_{j+i-1, k} G(x)^{k}\right] \\
& =\sum_{j=0}^{n-i} \sum_{k, r=0}^{\infty} \frac{n !}{(i-1) !(n-i) !}(-1)^{j}\left(\begin{array}{c}
n-i \\
j
\end{array}\right) \frac{b_{r+1}(r+1)}{r+k+1} d_{j+i-1, k} h_{r+k-1}(x) \\
& =\sum_{k, r=0}^{\infty} m_{k, r} h_{r+k-1}(x)
\end{aligned}
$$


where $h_{r+k-1}(x)$ is the exp-G density function with power parameter $r+k-1$ and

$$
m_{k, r}=\frac{n ! b_{r+1}(r+1)}{(i-1) !(r+k+1)} \sum_{j=0}^{n-i} \frac{(-1)^{j}}{j !(n-i-j) !} d_{j+i-1, k} .
$$

Moments of order statistics are more popular in reliability and quality control testing. Here, we provide two formulas for moments of order statistics of a TW-G distribution. The first one follows from moment of random variable $Y_{r, k}$ with the exp-G density function $h_{r+k-1}(x)$ as

$$
E\left(X_{i: n}^{p}\right)=\int_{-\infty}^{\infty} x^{p} f_{i: n}(x) d x=\sum_{k, r=0}^{\infty} m_{k, r} E\left(Y_{r, k}^{p}\right)
$$

We can present a second formula for $E\left(X_{i: n}^{p}\right)$ based on the quantile function $\mathrm{G}$ as

$$
E\left(X_{i: n}^{p}\right)=\sum_{k, r=0}^{\infty} m_{k, r}(r+k+1) \int_{-\infty}^{\infty} x^{p} g(x) G(x)^{r+k} d x=\sum_{k, r=0}^{\infty} m_{k, r}(r+k+1) \mathcal{L}(r, k),
$$

where $\mathcal{L}(r, k)=\int_{0}^{1}\left[G^{-1}(u)\right]^{p} u^{k+r} d u$. By similar calculations as those of ordinary moments, the integral $\mathcal{L}(r, k)$ for the TW-W, TW-L and TW-LL distributions can be expressed as

$$
\begin{aligned}
& \mathcal{L}(r, k)=\frac{1}{\lambda^{\frac{p}{\gamma}}} \sum_{l=0}^{k+r} \frac{(-1)^{l}}{(l+1)^{\frac{p}{\gamma}+1}} \Gamma\left(\frac{p}{\gamma}+1\right)\left(\begin{array}{c}
k+r \\
l
\end{array}\right), \\
& \mathcal{L}(r, k)=\frac{1}{\eta^{p}} \frac{\partial^{p}}{\partial t^{p}}(B(t+k+r+1,1-t))_{t=0}
\end{aligned}
$$

and

$$
\mathcal{L}(r, k)=\sum_{l=0}^{\infty} \frac{s^{p}\left(\frac{p}{c}\right)^{(l)}}{l !\left(l+k+r+\frac{p}{c}+1\right)},
$$

respectively.

\subsection{Unimodal Property}

Loosely speaking, the concept of unimodality is usually considered as a distribution with a density $f(x)$, which has one pick. Investigation of unimodal distributions originally started by Khinchin (1938). The main interest of unimodality is in optimization theory and mathematical programing. Also this property has become a useful tool in characterization of distributions appearing in different areas of statistic. In this section, we consider unimodal property of TW-G $(\alpha, \beta)$ distributions through their log-convex and log-concave properties. These will also enable as to investigate their hazard rate behaviour.

Theorem 1 Suppose that $g(x)$ is twice differentiable, then the pdf of a TW-G( $\alpha, \beta)$ distribution is log-convex if $\beta \leq 1$ and $g(x)$ is non-increasing and log-convex.

Proof. Since $g(x)$ is twice differentiable we have

$$
\frac{d^{2} \log (f(x))}{d x^{2}}=\frac{g^{\prime}(x)}{G(x)}\left[(\beta-1)-\alpha \beta G^{\beta}(x)\right]-(\beta-1) \frac{g^{2}(x)}{G^{2}(x)}\left[1+\alpha \beta G^{\beta}(x)\right]+\frac{d^{2} \log (g(x))}{d x^{2}} .
$$

Thus, $f(x)$ is log-convex when $\beta \leq 1$ and $g(x)$ is non-increasing and log-convex.

Theorem 2 Suppose that $g(x)$ is twice differentiable, then pdf of a TW-G( $\alpha, \beta)$ distribution is log-concave when $\beta \geq 1$ and either $G^{\beta}(x) \leq \frac{\beta-1}{\alpha \beta}$ and $g(x)$ is non-increasing and log-concave or $G^{\beta}(x) \geq \frac{\beta-1}{\alpha \beta}$ and $g(x)$ is non-decreasing and log-concave.

Proof. The proof is similar to that of Theorem 1.

Now, since log concave and log convex densities are unimodal, then we have

Corollary 1 Suppose that $g(x)$ is twice differentiable, then $T W-G(\alpha, \beta)$ distributions are unimodal if either $\beta \leq 1$ and $g(x)$ is non-increasing and log-convex or $\beta \geq 1$ and either $G^{\beta}(x) \leq \frac{\beta-1}{\alpha \beta}$ and $g(x)$ is non-increasing and log-concave or $G^{\beta}(x) \geq \frac{\beta-1}{\alpha \beta}$ and $g(x)$ is non-decreasing and log-concave. 
Consequently, since a log-concave (log-convex) pdf has non-decreasing (non-increasing) hazard function, we have the following.

Corollary 2 Suppose that $g(x)$ is twice differentiable, then $T W-G(\alpha, \beta)$ distributions have non-increasing hazard functions when $\beta \leq 1$ and $g(x)$ is non-increasing and log-convex and have non-decreasing hazard functions when $\beta \geq 1$ and either $G^{\beta}(x) \leq \frac{\beta-1}{\alpha \beta}$ and $g(x)$ is non-increasing and log-concave or $G^{\beta}(x) \geq \frac{\beta-1}{\alpha \beta}$ and $g(x)$ is non-decreasing and log-concave.

\subsection{Stochastic Orders}

One of the most fundamental measures of comparison of the behavior of random variables is stochastic ordering . Several types of stochastic orderings with different implications and applications can be seen in Shaked and Shanthikumar (2007). Here, we first recall definitions of the required stochastic orders: the likelihood ratio order $\left(\leq_{l r}\right)$, the usual stochastic $\operatorname{order}\left(\leq_{s t}\right)$, the hazard rate order $\left(\leq_{h r}\right)$, the reversed hazard rate order $\left(\leq_{r h}\right)$ and the expectation order $\left(\leq_{E}\right)$. Then, we shall stochastically compare TW- $\mathrm{G}(\alpha, \beta)$ and $\mathrm{B}-\mathrm{G}(\beta, \alpha)$ distributions. For instance, we shall reveal that, as lifetime distributions, B-G distribution has smaller reliability than TW-G distribution. Let $U$ and $V$ be two random variables with pdfs $f_{U}$ and $f_{V}$, respectively. Then, we have

i) $U \leq_{l r} V$ if $f_{V}(x) / f_{U}(x)$ is an increasing function in $\mathrm{x}$.

ii) $U \leq_{s t} V$ if $P(U \geq x) \leq P(V \geq x)$ for all $\mathrm{x}$.

iii) $U \leq_{h r} V$ if $f_{U}(x) / P(U \geq x) \leq f_{V}(x) / P(V \geq x)$ for all $\mathrm{x}$.

iv) $U \leq_{E} V$ if $E(U) \leq E(V)$.

Theorem 3 Let $U \sim B-G(\beta, \alpha)$ and $V \sim T W-G(\alpha, \beta)$. Then, $U \leq_{l r}\left(\leq_{h r}, \leq_{s t}\right.$ and $\left.\leq_{E}\right) V$ if $\alpha\left(1-\beta G(x)^{\beta-1}(1-G(x))\right) \geq 1$.

Proof. Since

it easily follows that

$$
\frac{f_{V}(x)}{f_{U}(x)}=\frac{\alpha \beta B(\beta, \alpha) \exp \left(-\alpha G(x)^{\beta}\right)}{\left(1-e^{-\alpha}\right)(1-G(x))^{\alpha-1}},
$$

$$
\frac{d}{d x}\left(\frac{f_{V}(x)}{f_{U}(x)}\right)=\frac{\alpha \beta B(\beta, \alpha) g(x) \exp \left(-\alpha G(x)^{\beta}\right)(1-G(x))^{\alpha-2}\left[-\alpha \beta G(x)^{\beta-1}(1-G(x))+(\alpha-1)\right]}{\left(1-e^{-\alpha}\right)(1-G(x))^{2 \alpha-2}},
$$

which is non-negative, if $\alpha\left[1-\beta G(x)^{\beta-1}(1-G(x))\right] \geq 1$. Therefore, by (i) $U \leq_{l r} V$ if $\alpha\left[1-\beta G(x)^{\beta-1}(1-G(x))\right] \geq 1$. Now, based on the results of Shaked and Shanthikumar (2007) that: $U \leq_{l r} V \Rightarrow U \leq_{h r} V \Rightarrow_{U} \leq_{s t} V \Rightarrow_{U} \leq_{E} V$, we have the results if $\alpha\left[1-\beta G(x)^{\beta-1}(1-G(x))\right] \geq 1$.

\subsection{Stress-strength Reliability Function}

Let $X_{1} \sim T W-G\left(\alpha_{1}, \beta_{1}, \xi\right)$ and $X_{2} \sim T W-G\left(\alpha_{2}, \beta_{2}, \xi\right)$ be independent random variables with pdf's and cdf's $f_{1}, F_{1}$ and $f_{2}, F_{2}$, respectivly, and $\xi$ be the vector of parameters of the parent distribution $G$. Then, the stress-strength reliability function $R=P\left(X_{1}>X_{2}\right)$ is given by

$$
R=P\left(X_{1}>X_{2}\right)=\int_{0}^{\infty} F_{X_{2}}(x) f_{X_{1}}(x) d x .
$$

Thus, by Eqs. (11) and (12), we have

$$
\begin{aligned}
R & =\sum_{k, s=0}^{\infty} b_{k}^{(2)} b_{s+1}^{(1)} \int_{-\infty}^{\infty} F_{k}^{*}(x) f_{s+1}^{*}(x) d x=\sum_{k, s=0}^{\infty} b_{k}^{(2)} b_{s+1}^{(1)} \int_{-\infty}^{\infty} G(x)^{k+s}(s+1) g(x) d x, \\
& =\sum_{k, s=0}^{\infty} b_{k}^{(2)} b_{s+1}^{(1)} \frac{s+1}{k+s+1},
\end{aligned}
$$

where $b_{s+1}^{(1)}$ and $b_{k}^{(2)}$ are

$$
\begin{aligned}
b_{s+1}^{(1)} & =\sum_{i=1}^{\infty} \sum_{j=s+1}^{\infty} \frac{(-1)^{i+j+s+1} \alpha_{1}{ }^{i}}{i !\left(1-\exp \left(-\alpha_{1}\right)\right)}\left(\begin{array}{c}
i \beta_{1} \\
j
\end{array}\right)\left(\begin{array}{c}
j \\
s+1
\end{array}\right), \\
b_{k}^{2} & =\sum_{i=1}^{\infty} \sum_{j=k}^{\infty} \frac{(-1)^{i+j+k} \alpha_{2}{ }^{i}}{i !\left(1-\exp \left(-\alpha_{2}\right)\right)}\left(\begin{array}{c}
i \beta_{2} \\
j
\end{array}\right)\left(\begin{array}{l}
j \\
k
\end{array}\right),
\end{aligned}
$$

Clearly, if $\alpha_{1}=\alpha_{2}, \beta_{1}=\beta_{2}$, Eq. (38) reduces to $R=\frac{1}{2}$. 


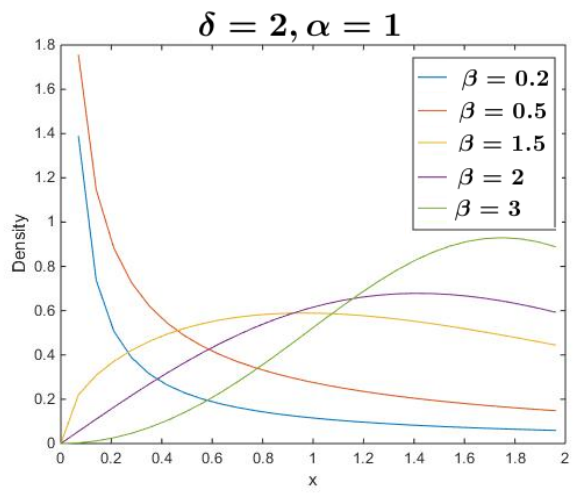

(a)

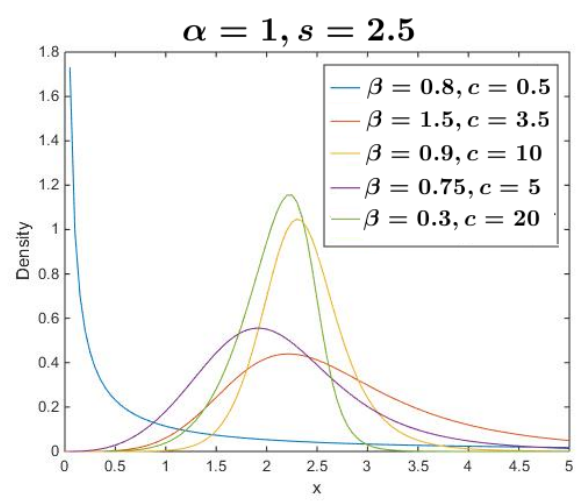

(c)

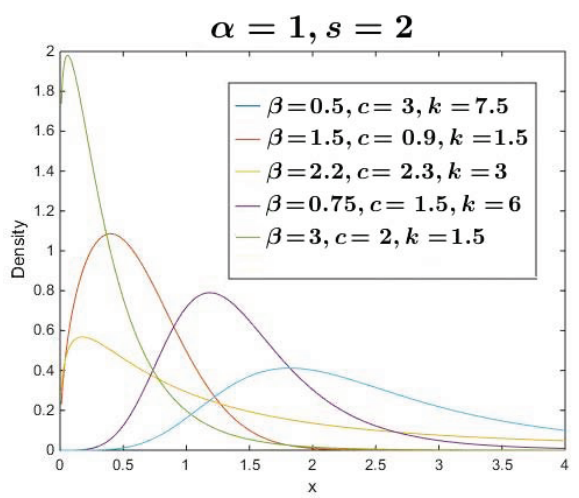

(e)

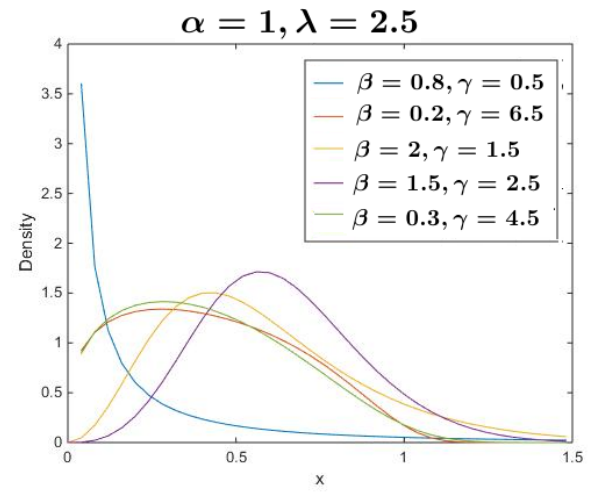

(b)

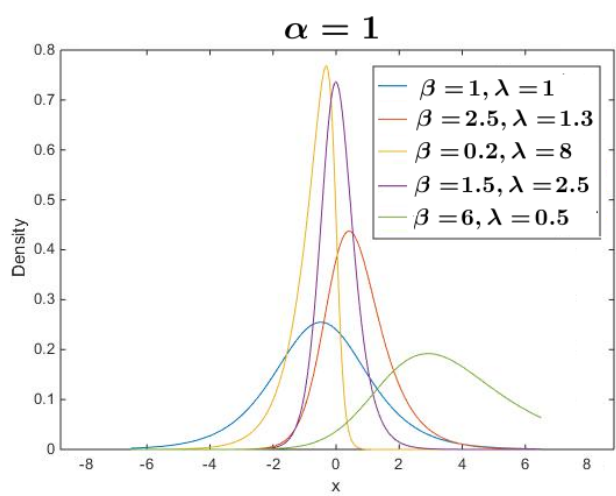

(d)

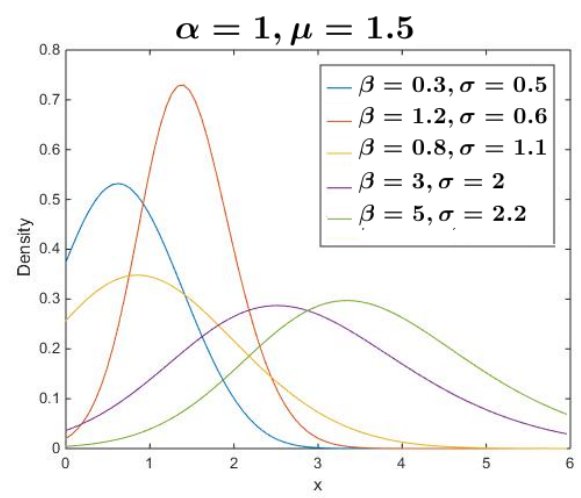

(f)

Figure 1. Plots of the (a) TW-U (b) TW-W (c) TW-LL (d) TW-L (e) TW-BXII and (f) TW-N densities.

\section{Shapes of the pdf and hrf}

Figures 1 and 2 indicate possible shapes of the pdfs and hrfs, respectively, of TW-G distributions for its various parameter values. As we observe, Figure 1 shows that the TW-G family distributions have various shapes such as symmetrical, left and right skewed, bathtub and reversed-J. Figure 2, also shows that hazard rate of the new distributions have great flexibility such as decreasing, increasing, bathtub, upside-down bathtub, J, reversed- J and S shapes. Hence, TW-G distributions are useful for fitting to different data sets with various shapes. Infact, shapes of the pdfs and hr functions can also be expressed analytically. The critical points of the NW-G density are the roots of the following equation:

$$
\frac{g(x)}{G(x)}(\beta-1)-\alpha \beta G(x)^{\beta-1} g(x)+\frac{g^{\prime}(x)}{g(x)}=0,
$$

and critical points of $h(x)$ are obtained from the equation:

$$
(\beta-1) \frac{g(x)}{G(x)}-\alpha \beta G^{\beta-1}(x) g(x)+\frac{g^{\prime}(x)}{g(x)}-\frac{\alpha \beta G^{\beta-1}(x) g(x) e^{-\alpha G^{\beta}(x)}}{e^{-\alpha G^{\beta}(x)}-e^{-\alpha}}=0 .
$$




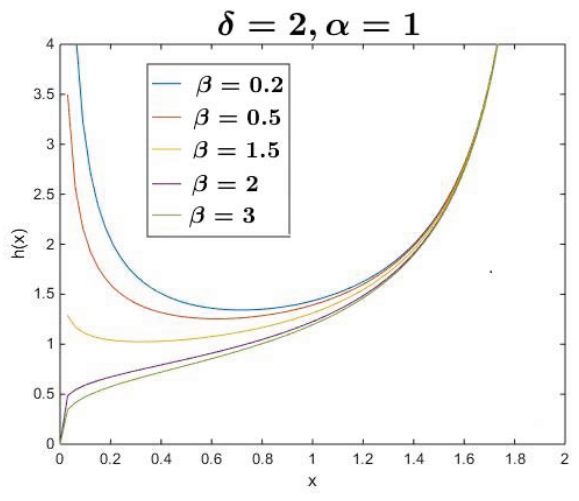

(a)

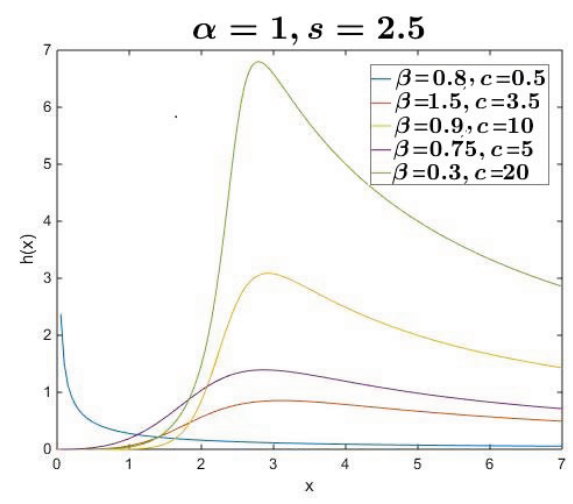

(c)

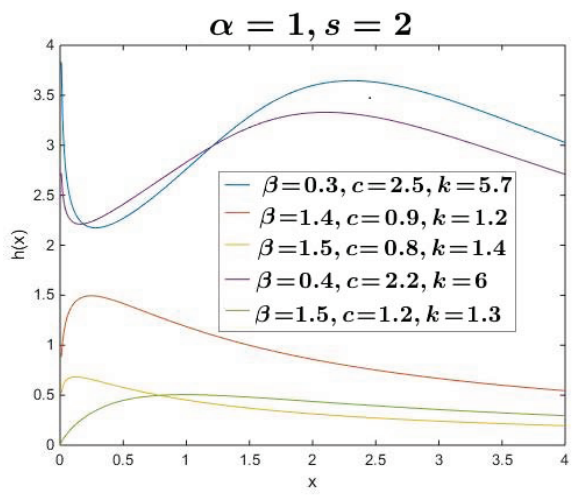

(e)

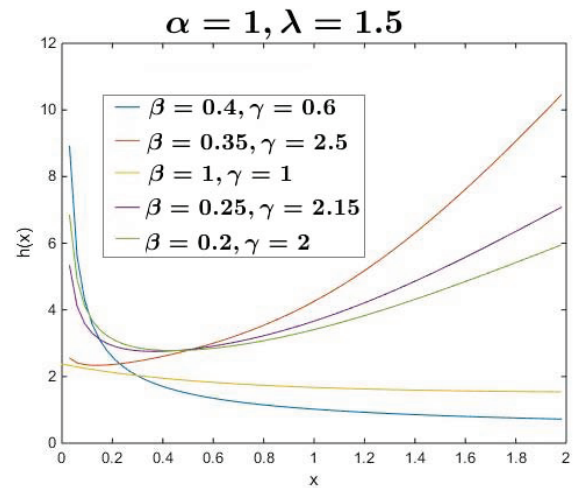

(b)

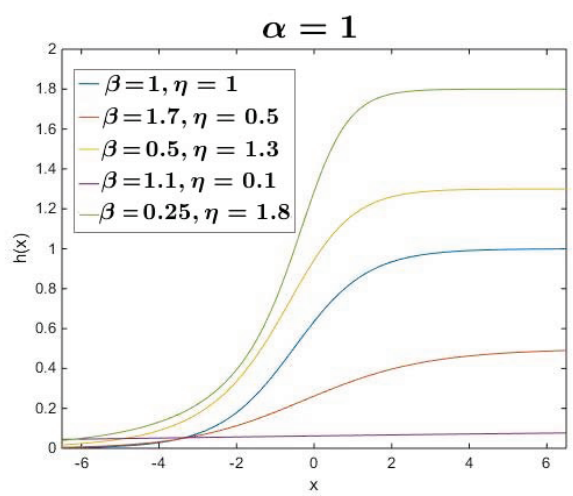

(d)

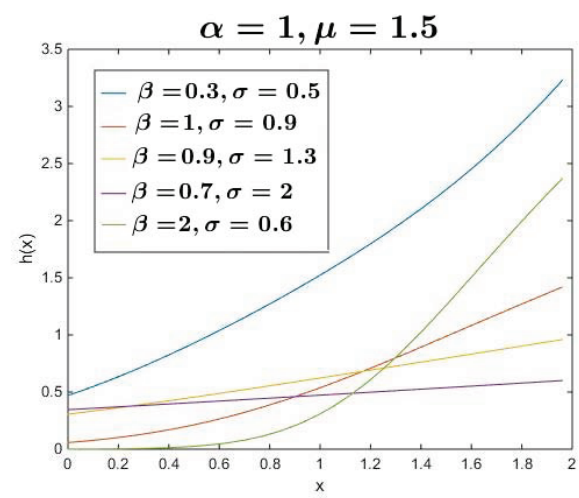

(f)

Figure 2. Plots of the (a) TW-U (b) TW-W (c) TW-LL (d) TW-L (e) TW-BXII and (f) TW-N hazard rates.

By using most symbolic computation software platforms, we can examine Eqs. (39) and (40) to determine the local maxima and minima and inflexion points.

\section{Maximum Likelihood Estimation(MLE)}

In this section, we discuss estimation of the parameters of TW-G model by the maximum likelihood method. Let $x_{1}, \ldots, x_{n}$ be $n$ observations of a random sample from a TW-G distribution with parameters $\Theta=(\alpha, \beta, \xi)^{T}$, where $\xi$ is a $K \times 1$ parameter vector in $G($.$) . The log-likelihood function for parameters \Theta=(\alpha, \beta, \xi)^{T}$ is given by

$$
\begin{aligned}
\ell_{n}(\Theta) & =n \log \alpha+n \log \beta+\sum_{i=1}^{n} \log g\left(x_{i}\right)+(\beta-1) \sum_{i=1}^{n} \log G\left(x_{i}\right) \\
& -\alpha \sum_{i=1}^{n} G^{\beta}\left(x_{i}\right)-n \log \left(1-e^{-\alpha}\right) .
\end{aligned}
$$


The first derivatives of $\ell_{n}(\Theta)$ with respect to the parameters $\alpha, \beta$ and $\xi$ are:

$$
\begin{gathered}
\frac{\partial \ell_{n}(\Theta)}{\partial \alpha}=\frac{n}{\alpha}-\sum_{i=1}^{n} G^{\beta}\left(x_{i}\right)-\frac{n e^{-\alpha}}{1-e^{-\alpha}}=0, \\
\frac{\partial \ell_{n}(\Theta)}{\partial \beta}=\frac{n}{\beta}+\sum_{i=1}^{n} \log G\left(x_{i}\right)-\alpha \beta \sum_{i=1}^{n} g\left(x_{i}\right) G^{\beta-1}\left(x_{i}\right)=0, \\
\frac{\partial \ell_{n}(\Theta)}{\partial \xi_{j}}=\sum_{i=1}^{n} \frac{\dot{g}(x)}{g\left(x_{i}\right)}+(\beta-1) \sum_{i=1}^{n} \frac{\dot{G}\left(x_{i}\right)}{G\left(x_{i}\right)}-\alpha \beta \sum_{i=1}^{n} G(x)^{\beta-1} \dot{G}\left(x_{i}\right)=0, \quad j=1,2, \ldots, k,
\end{gathered}
$$

where $\dot{g}(x)=\frac{\partial g\left(x_{i}\right)}{\partial \xi_{j}}$ and $\dot{G}\left(x_{i}\right)=\frac{\partial G\left(x_{i}\right)}{\partial \xi_{j}}$. Therefore, solutions of these equations yeild the MLE of $\Theta$ parameters, which can be obtioned by a numerical method. For interval estimation and hypothesis testings on the parameters, we need the information matrix $I(\theta)=E\left[-\frac{\partial^{2} \ell_{n}(\Theta)}{\partial \Theta^{2}}\right]$, such that

$$
\frac{\partial^{2} \ell_{n}(\Theta)}{\partial \Theta^{2}}=\left[\begin{array}{lll}
I_{11} & I_{12} & I_{13} \\
I_{12} & I_{22} & I_{23} \\
I_{13} & I_{23} & I_{33}
\end{array}\right],
$$

whose elements are

$$
\begin{aligned}
I_{11}=\frac{\partial^{2} \ell_{n}(\Theta)}{\partial \alpha^{2}}=-\frac{n}{\alpha^{2}}+\frac{n e^{-\alpha}}{\left(1-e^{-\alpha}\right)^{2}}, \\
I_{12}=\frac{\partial^{2} \ell_{n}(\Theta)}{\partial \alpha \partial \beta}=\sum_{i=0}^{n} g\left(x_{i}\right) G^{\beta-1}\left(x_{i}\right), \\
I_{22}=\frac{\partial^{2} \ell_{n}(\Theta)}{\partial \beta^{2}}=-\frac{n}{\beta^{2}}-\alpha \sum_{i=1}^{n} g\left(x_{i}\right) G^{\beta-1}\left(x_{i}\right)-\alpha \beta(\beta-1) \sum_{i=1}^{n} g^{2}\left(x_{i}\right) G^{\beta-2}\left(x_{i}\right), \\
I_{13}=\frac{\partial^{2} \ell_{n}(\Theta)}{\partial \alpha \partial \xi_{j}}=\sum_{i=1}^{n} G^{\beta-1}\left(x_{i}\right) \dot{G}\left(x_{i}\right), \\
I_{23}=\frac{\partial^{2} \ell_{n}(\Theta)}{\partial \beta \partial \xi_{j}}=\sum_{i=1}^{n} \frac{\dot{G}\left(x_{i}\right)}{G\left(x_{i}\right)}-\alpha \sum_{i=1}^{n} G^{\beta-1}\left(x_{i}\right) \dot{G}\left(x_{i}\right)-\alpha \beta(\beta-1) \sum_{i=1}^{n} g\left(x_{i}\right) G^{\beta-2}\left(x_{i}\right) \dot{G}\left(x_{i}\right), \\
I_{33}=\frac{\partial^{2} \ell_{n}(\Theta)}{\partial \xi_{j}^{2}}=\sum_{i=1}^{n} \frac{\ddot{g}\left(x_{i}\right) g\left(x_{i}\right)-\left(\dot{g}\left(x_{i}\right)\right)^{2}}{g^{2}\left(x_{i}\right)}+(\beta-1) \sum_{i=1}^{n} \frac{\ddot{G}\left(x_{i}\right) G\left(x_{i}\right)-\left(\dot{G}\left(x_{i}\right)\right)^{2}}{G^{2}\left(x_{i}\right)} \\
-\alpha \beta(\beta-1) \sum_{i=1}^{n} G^{\beta-2}\left(x_{i}\right)\left(\dot{G}\left(x_{i}\right)\right)^{2}-\alpha \beta \sum_{i=1}^{n} G^{\beta-1}\left(x_{i}\right) \ddot{G}\left(x_{i}\right),
\end{aligned}
$$

where $\ddot{g}\left(x_{i}\right)=\frac{\partial^{2} g\left(x_{i}\right)}{\partial \xi_{j}^{2}}$ and $\ddot{G}\left(x_{i}\right)=\frac{\partial^{2} G\left(x_{i}\right)}{\partial \xi_{j}^{2}}$ for $j=1,2, \ldots, k$. If MLE of $\Theta=(\alpha, \beta, \xi)$ is $\hat{\Theta}=(\hat{\alpha}, \hat{\beta}, \hat{\xi})$, then by assuming the regularity conditions, $(\hat{\alpha}-\alpha, \hat{\beta}-\beta, \hat{\xi}-\xi)$ converges in distribution to $(\mathrm{k}+2)$ multivariate normal distribution with zero means and variance-covariance matrix $I^{-1}$.

\section{Application}

In this section, we apply an data set corresponding to remission times of a random sample of 128 bladder cancer patients (cf. Lee \& Wang, 2003), to provide an application of six submodels of the TW-G distribution, i.e., TW-U, TW-W, TW-LL, TW-L, TW-BXII and TW-N distributions. The data are given below:

$0.08,2.09,3.48,4.87,6.94,8.66,13.11,23.63,0.20,2.23,3.52,4.98,6.97,9.02,13.29,0.40,2.26,3.57,5.06,7.09,9.22$, $13.80,25.74,0.50,2.46,3.64,5.09,7.26,9.47,14.24,25.82,0.51,2.54,3.70,5.17,7.28,9.74,14.76,26.31,0.81,2.62$, $3.82,5.32,7.32,10.06,14.77,32.15,2.64,3.88,5.32,7.39,10.34,14.83,34.26,0.90,2.69,4.18,5.34,7.59,10.66,15.96$, $36.66,1.05,2.69,4.23,5.41,7.62,10.75,16.62,43.01,1.19,2.75,4.26,5.41,7.63,17.12,46.12,1.26,2.83,4.33,5.49$, $7.66,11.25,17.14,79.05,1.35,2.87,5.62,7.87,11.64,17.36,1.40,3.02,4.34,5.71,7.93,11.79,18.10,1.46,4.40,5.85$, $8.26,11.98,19.13,1.76,3.25,4.50,6.25,8.37,12.02,2.02,3.31,4.51,6.54,8.53,12.03,20.28,2.02,3.36,6.76,12.07$, $21.73,2.07,3.36,6.93,8.65,12.63,22.69$.

Table 1 indicates the MLEs of the parameters, Akaike Information Criterion (AIC) as AIC $=-2 \log L+2 k$, the Bayesian information criterion (BIC) as $B I C=-2 \log L+k \log (n)$, 
Table 1. MLEs of the parameters, AIC, BIC, HGIC and K-S (p-value) of the TW-U, TW-W, TW-L, TW-LL, TW-BXII and TW-N models

\begin{tabular}{cccccc}
\hline Model & MLEs of parameters & AIC & BIC & HQIC & K-S (p-value) \\
\hline TW-U & $(\hat{\alpha}, \hat{\beta}, \hat{\delta})=(9.123,1.047,79.050)$ & 854.86 & 863.41 & 858.33 & $0.070(0.555)$ \\
TW-W & $(\hat{\alpha}, \hat{\beta}, \hat{\lambda}, \hat{\gamma})=(3.740,1.602,4.051,16.306)$ & 827.44 & 838.85 & 832.08 & $0.030(0.994)$ \\
TW-L & $(\hat{\alpha}, \hat{\beta}, \hat{\eta})=(2.422,5.144,0.160)$ & 873.32 & 881.87 & 876.79 & $0.101(0.145)$ \\
TW-LL & $(\hat{\alpha}, \hat{\beta}, \hat{s}, \hat{c})=(0.011,0.585,2.173,9.251)$ & 827.39 & 838.80 & 832.03 & $0.032(0.999)$ \\
TW-BXII & $(\hat{\alpha}, \hat{\beta}, \hat{s}, \hat{c}, \hat{k})=(0.009,0.720,1.352,1.352,10.106)$ & 829.29 & 843.55 & 835.09 & $0.030(0.998)$ \\
TW-N & $(\hat{\alpha}, \hat{\beta}, \hat{\mu}, \hat{\sigma})=(4.247,5.097,0.09,15.367)$ & 907.05 & 918.46 & 911.69 & $0.128(0.031)$ \\
\hline
\end{tabular}

HannanCQuinn information criterion (HQIC) as HQIC $=-2 L+2 k \log (\log (n))$, Kolmogorov CSmirnov (KCS) distance between the empirical distribution and the fitted model (and its corresponding P-value in parentheses), where $\mathrm{n}$ is the number of observations, $k$ is number of parameters in the model and $L$ is the maximized value of the likelihood function, for all six special models of the TW-G distribution. Analysis of Table 1 indicates that TW-LL distribution has the lowest AIC, BIC, HQIC and K-S values, and so it is the best model to fit to this data. We shall also arrive at the same results by comparing $\mathrm{p}$-values for all six models.

Table 2 illustrate AIC, BIC and HQIC for the three submodels of B-G distributions. Comparing Tables 1 and 2 indicate that TW-W and TW-BXII distributions provide better fits in comparison with B-W and B-BXII distributions. The histogram of the data and fitted TW-U, TW-W, TW-L, TW-BXII, TW-LL and TW-N distributions are plotted in Figure 3. As we observe, the TW-U, TW-W, TW-LL and TW-BXII models yield better fits among other models. The empirical and fitted survival functions of TW-LL distribution are shown in Figure 4.

Table 2. MLEs of the parameters, AIC, BIC, HGIC of the B-W, B-LL, B-BXII models

\begin{tabular}{ccccc}
\hline Model & MLEs of parameters & AIC & BIC & HQIC \\
\hline B-W & $(\hat{\beta}, \hat{\alpha}, \hat{\lambda}, \hat{\gamma})=(0.907,2.735,0.469,0.666)$ & 829.36 & 838.85 & 832.08 \\
B-LL & $(\hat{\beta}, \hat{\alpha}, \hat{s}, \hat{c})=(1.344,0.720,10.214,1.827)$ & 827.39 & 838.80 & 832.03 \\
B-BXII & $(\hat{\beta}, \hat{\alpha}, \hat{s}, \hat{c}, \hat{k})=(0.268,0.796,9.653,1.678,5.667)$ & 829.32 & 843.58 & 835.11 \\
\hline
\end{tabular}

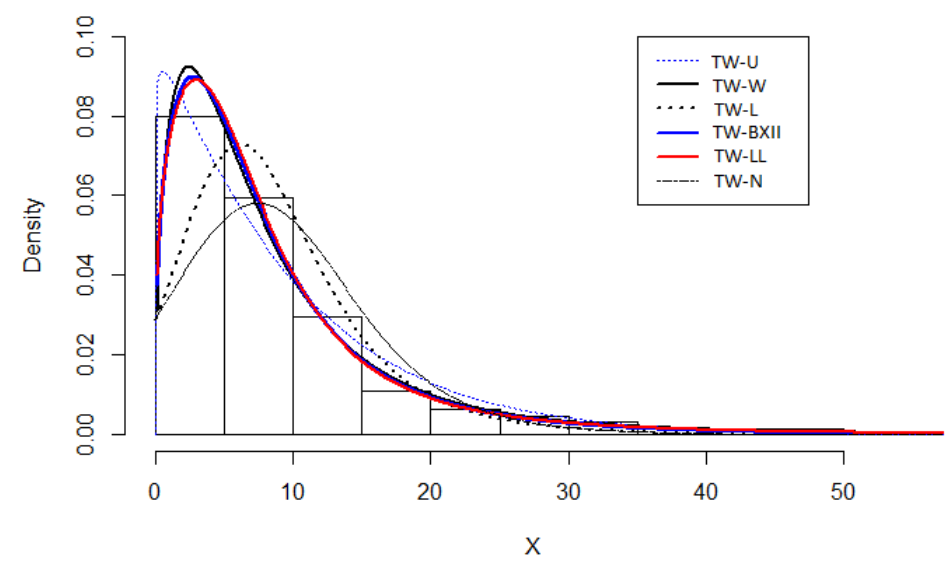

Figure 3. The histogram of remission times of bladder cancer data and the fitted pdfs of TW-U, TW-W, TW-L, TW-BXII, TW-LL and TW-N. 


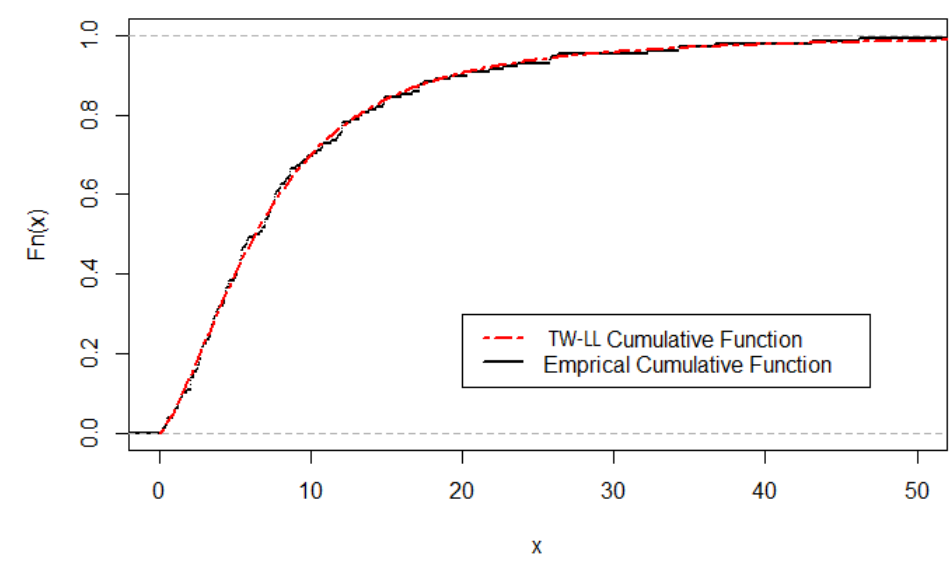

Figure 4. The empirical and fitted survival functions of TW-LL distribution.

\section{Acknowledgements}

The first author is grateful to the Graduate Office of the University of Isfahan for their support.

\section{References}

Barreto-Souza, W., Santos, A. H., \& Cordeiro, G. M. (2010). The beta generalized exponential distribution. Journal of Statistical Computation and Simulation, 80(2), 159-172. http://dx.doi.org/10.1080/00949650802552402.

Cooray, K. (2010). Generalized Gumbel distribution. Journal of Applied Statistics, 37(1), 171-179. http://dx.doi.org/10.1080/02664760802698995.

Cordeiro, G. M., Castellares, F., Montenegro, L. C., \& de Castro, M. (2013). The beta generalized gamma distribution. Statistics, 47(4), 888-900. http://dx.doi.org/10.1080/02331888.2012.658397.

Cordeiro, G. M., \& Nadarajah, S. (2011). Closed-form expressions for moments of a class of beta generalized distributions. Brazilian Journal of Probability and Statistics, 25, 14C33. http://dx.doi:10.1214/09-BJPS109

Cordeiro, G. M., Silva, G. O., \& Ortega, E. M., (2013). The beta-Weibull geometric distribution. Statistics, 47(4), 817-834. http://dx.doi.org/10.1080/02331888.2011.577897.

Eugene, N., Lee, C., \& Famoye, F. (2002). Beta-normal distribution and its applications. Communications in StatisticsCTheory and Methods, 31, 497C512. http://dx.doi.org/10.1081/STA-120003130

Gradshteyn, I. S., \& Ryzhik, I. M. (2007). Table of Integrals, Series, and Products (seventh ed.) Academic Press, San Diego. http://dx.doi.org/10.1118/1.594867

Gupta, R. D., \& Kundu, D. (2001). Exponentiated exponential family: an alternative to gamma and Weibull distributions. Biometrical journal, 43(1), 117-130. http://dx.doi.org/10.1002/1521-4036

Gupta, R. D., \& Kundu, D. (1999). Theory and methods: Generalized exponential distributions. Australian and New Zealand Journal of Statistics, 41(2), 173-188. http://dx.doi.org/10.1111/1467-842X.00072

Handique, L., \& Chakraborty, S. (2016). The Beta Generalized Marshall-Olkin-G Family of Distributions. arXiv preprint arXiv:1608.05985.

Khinchin, A. Ya. (1938).On unimodal distributions. Research Institute for Mathematical Sciences (in Russian), University of Tomsk, 2(2), $1 \mathrm{C} 7$.

Lee, C., Famoye, F., \& Olumolade, O. (2007). Beta-Weibull distribution: some properties and applications to censored data. Journal of modern applied statistical methods, 6(1), 17. http://dx.doi.org/10.22237/jmasm/1177992960.

Lee, E. T., \& Wang, J. W. (2003). Statistical Methods for Survival Data Analysis (3rd ed.) Wiley, NewYork. http:// dx.doi.org/10.1002/0471458546

MirMostafaee, S. M. T. K., Mahdizadeh, M., \& Nadarajah, S. (2015). The beta Lindley distribution. Journal of Data Science, 13(3), 603-625. 
Mudholkar, G. S., Srivastava, D. K., \& Freimer, M. (1995). The exponentiated Weibull family: A reanalysis of the bus-motor failure data. Technometrics, 37, 436C445. http://dx.doi.org/10.2307/1269735

Nadarajah, S. (2005a). Exponentiated beta distributions. Computers and Mathematics with Applications, 49(7), 10291035. https://doi.org/10.1016/j.camwa.2004.11.008./02331880500065488.

Nadarajah, S., \& Kotz, S. (2006). The beta exponential distribution. Reliability engineering and system safety, 91(6), 689-697. https://doi.org/10.1016/j.ress.2005.05.008.

Nadarajah, S., \& Kotz, S. (2004). The beta Gumbel distribution. Mathematical Problems in Engineering, (4), 323-332. http://dx.doi.org/10.1155/S1024123X04403068.

Nassar, M. M., \& Eissa, F. H. (2003). On the exponentiated Weibull distribution. Communications in Statistics-Theory and Methods, 32(7), 1317-1336. http://dx.doi.org/10.1081/STA-120021561.

Silva, G. O., Ortega, E. M., \& Cordeiro, G. M. (2010). The beta modified Weibull distribution. Lifetime data analysis, 16(3), 409-430. http://dx.doi.org/10.1007/s10985-010-9161-1.

\section{Copyrights}

Copyright for this article is retained by the author(s), with first publication rights granted to the journal.

This is an open-access article distributed under the terms and conditions of the Creative Commons Attribution license (http://creativecommons.org/licenses/by/4.0/). 